\title{
Confinement of spinless particles by Coulomb potentials in two-dimensional space-time
}

\author{
Antonio S. de Castro \\ UNESP - Campus de Guaratinguetá \\ Departamento de Física e Química \\ Caixa Postal 205 \\ 12516-410 Guaratinguetá SP - Brasil \\ Electronic mail: castro@feg.unesp.br
}




\begin{abstract}
The problem of confinement of spinless particles in $1+1$ dimensions is approached with a linear potential by considering a mixing of Lorentz vector and scalar couplings. Analytical bound-states solutions are obtained when the scalar coupling is of sufficient intensity compared to the vector coupling.
\end{abstract}




\section{Introduction}

The potential generated by a point charge, the Coulomb potential, depends on the dimensionality of space and in a $1+1$ dimension it is linear (see, e.g. [1]). As the time component of a Lorentz vector, the $1+1$ dimensional Coulomb potential is linear and so it provides a constant electric field always pointing to, or from, the point charge. This problem is related to the confinement of fermions in the Schwinger and in the massive Schwinger models [2]- 3 ] and in the Thirring-Schwinger model [4. It is frustrating that, due to the tunneling effect (Klein's paradox), there are no bound states for this kind of potential regardless of the strength of the potential [5]-[6]. The linear potential, considered as a Lorentz scalar, is also related to the quarkonium model in one-plus-one dimensions [7]- [8]. Recently it was incorrectly concluded that even in this case there is just one bound state [9]. Later, the proper solutions for this last problem were found [10]-12. However, it is well known from the quarkonium phenomenology in the real $3+1$ dimensional world that the best fit for meson spectroscopy is found for a convenient mixture of vector and scalar potentials put by hand in the equations (see, e.g., [13). The same can be said about the treatment of the nuclear phenomena describing the influence of the nuclear medium on the nucleons [14- 27]. The mixed vector-scalar potential has also been analyzed with the Dirac equation in $1+1$ dimensions for a linear potential [28] as well as for a general potential which goes to infinity as $|x| \rightarrow \infty$ [29]. In both of those last references it has been concluded that there is confinement if the scalar coupling is of sufficient intensity compared to the vector coupling.

Since the pure vector Coulomb potential also frustrates the existence of bound-state solutions in the Klein-Gordon (KG) equation [6], the present work proposes to consider a general mixing of vector and scalar Lorentz structures. Such as in the case of the Dirac equation, this sort of mixing shows to be a powerful tool to obtain a deeper insight about the nature of the KG equation and its solutions. The problem is mapped into an exactly solvable Sturm-Liouville problem of a Schrödinger-like equation with an effective harmonic oscillator potential. In two very special circumstantes the effective potential becomes a Coulomb potential and a sort of phase transition shows up resulting in a spectrum only consisting exclusively of either particle-energy levels or antiparticle-energy levels. 


\section{The Klein-Gordon equation with mixed vector- scalar potentials in a $1+1$ dimension}

In the presence of vector and scalar potentials the $1+1$ dimensional timeindependent $\mathrm{KG}$ equation for a spinless particle of rest mass $m$ reads

$$
-\hbar^{2} c^{2} \frac{d^{2} \psi}{d x^{2}}+\left(m c^{2}+V_{s}\right)^{2} \psi=\left(E-V_{v}\right)^{2} \psi
$$

where $E$ is the energy of the particle, $c$ is the velocity of light and $\hbar$ is the Planck constant. The vector and scalar potentials are given by $V_{v}$ and $V_{s}$, respectively. The subscripts for the terms of potential denote their properties under a Lorentz transformation: $v$ for the time component of the 2-vector potential and $s$ for the scalar term. It is worth to note that the KG equation is covariant under $x \rightarrow-x$ if $V_{v}(x)$ and $V_{s}(x)$ remain the same. Also note that $\psi$ remains invariant under the simultaneous transformations $E \rightarrow-E$ and $V_{v} \rightarrow-V_{v}$. Furthermore, for $V_{v}=0$, the case of a pure scalar potential, the negative- and positive-energy levels are disposed symmetrically about $E=0$.

The KG equation can also be written as

$$
H_{e f f} \psi=-\frac{\hbar^{2}}{2 m} \psi^{\prime \prime}+V_{e f f} \psi=E_{e f f} \psi
$$

where

$$
E_{e f f}=\frac{E^{2}-m^{2} c^{4}}{2 m c^{2}}, \quad V_{e f f}=\frac{V_{s}^{2}-V_{v}^{2}}{2 m c^{2}}+V_{s}+\frac{E}{m c^{2}} V_{v}
$$

From this one can see that for potentials which tend to $\pm \infty$ as $|x| \rightarrow \infty$ the KG equation furnishes a purely discrete spectrum for $\left|V_{s}\right|>\left|V_{v}\right|$, or for $\left|V_{s}\right|=\left|V_{v}\right|$ and $V_{s}+V_{v} E /\left(m c^{2}\right)>0$. On the other hand, if the potentials vanish as $|x| \rightarrow \infty$ the continuum spectrum is omnipresent but the necessary conditions for the existence of a discrete spectrum is not an easy task for general functional forms. The boundary conditions on the eigenfunctions come into existence by demanding that the effective Hamiltonian given (2) is Hermitian, viz.

$$
\int_{a}^{b} d x \psi_{n}^{*}\left(H_{e f f} \psi_{n^{\prime}}\right)=\int_{a}^{b} d x\left(H_{e f f} \psi_{n}\right)^{*} \psi_{n^{\prime}}
$$


where $\psi_{n}$ is an eigenfunction corresponding to an effective eigenvalue $\left(E_{\text {eff }}\right)_{n}$ and $(a, b)$ is the interval under consideration. In passing, note that a necessary consequence of Eq. (44) is that the eigenfunctions corresponding to distinct effective eigenvalues are orthogonal. It can be shown that (4) is equivalent to

$$
\left[\psi_{n}^{*} \frac{d \psi_{n^{\prime}}}{d x}-\frac{d \psi_{n}^{*}}{d x} \psi_{n^{\prime}}\right]_{x=a}^{x=b}=0
$$

In the nonrelativistic approximation (potential energies small compared to $m c^{2}$ and $\left.E \simeq m c^{2}\right)$ Eq. (11) becomes

$$
\left(-\frac{\hbar^{2}}{2 m} \frac{d^{2}}{d x^{2}}+V_{v}+V_{s}\right) \psi=\left(E-m c^{2}\right) \psi
$$

so that $\psi$ obeys the Schrödinger equation with binding energy equal to $E-$ $m c^{2}$ without distinguishing the contributions of vector and scalar potentials.

It is remarkable that the $\mathrm{KG}$ equation with a scalar potential, or a vector potential contaminated with some scalar coupling, is not invariant under $V \rightarrow V+$ const., this is so because only the vector potential couples to the positive-energies in the same way it couples to the negative-ones, whereas the scalar potential couples to the mass of the particle. Therefore, if there is any scalar coupling the absolute values of the energy will have physical significance and the freedom to choose a zero-energy will be lost. It is well known that a confining potential in the nonrelativistic approach is not confining in the relativistic approach when it is considered as a Lorentz vector. It is surprising that relativistic confining potentials may result in nonconfinement in the nonrelativistic approach. This last phenomenon is a consequence of the fact that vector and scalar potentials couple differently in the KG equation whereas there is no such distinction among them in the Schrödinger equation. This observation permit us to conclude that even a "repulsive" potential can be a confining potential. The case $V_{v}=-V_{s}$ presents bounded solutions in the relativistic approach, although it reduces to the free-particle problem in the nonrelativistic limit. The attractive vector potential for a particle is, of course, repulsive for its corresponding antiparticle, and vice versa. However, the attractive (repulsive) scalar potential for particles is also attractive (repulsive) for antiparticles. For $V_{v}=V_{s}$ and an attractive vector potential for particles, the scalar potential is counterbalanced by the vector potential for antiparticles as long as the scalar potential is attractive and the vector 
potential is repulsive. As a consequence there is no bounded solution for antiparticles. For $V_{v}=0$ and a pure scalar attractive potential, one finds energy levels for particles and antiparticles arranged symmetrically about $E=0$. For $V_{v}=-V_{s}$ and a repulsive vector potential for particles, the scalar and the vector potentials are attractive for antiparticles but their effects are counterbalanced for particles. Thus, recurring to this simple standpoint one can anticipate in the mind that there is no bound-state solution for particles in this last case of mixing. For short, when $V_{v}= \pm V_{s}$ the spectrum only consists of energy levels either for particles or for antiparticles.

\section{The mixed vector-scalar Coulomb poten- tial}

Now let us focus our attention on scalar and vector potentials in the form

$$
V_{s}=g_{s}|x|, \quad V_{v}=g_{v}|x|
$$

where the coupling constants, $g_{s}$ and $g_{v}$, are real parameters. In this case the second equation of (3) transmutes into

$$
V_{e f f}=\frac{1}{2} A x^{2}+B|x|
$$

where

$$
A=\frac{g_{s}^{2}-g_{v}^{2}}{m c^{2}}, \quad B=g_{s}+\frac{E}{m c^{2}} g_{v}
$$

Therefore, one has to search for bounded solutions in an effective shifted harmonic oscillator potential for $g_{s}^{2} \neq g_{v}^{2}$, or Coulomb potential for $g_{s}^{2}=g_{v}^{2}$. The KG eigenvalues are obtained by inserting the effective eigenvalues into the first equation of (3). Since the effective potential is even under $x \rightarrow$ $-x$, the KG eigenfunction can be expressed as a function of definite parity. Thus, we can concentrate our attention on the positive half-line and impose boundary conditions on $\psi$ at $x=0$ and $x=+\infty$. From (15) one can see that in addition to $\psi(\infty)=0$, the boundary conditions at the origin can be met in two distinct ways: odd functions obey the Neumann condition $\left(d \psi /\left.d x\right|_{x=0}=\right.$

$0)$ whereas even functions obey the Dirichlet condition $(\psi(0)=0)$.

Now we move to consider a quantitative treatment of our problem by considering the two distinct classes of effective potentials. 


\subsection{The effective shifted harmonic oscillator potential $\left(g_{s}^{2} \neq g_{v}^{2}\right)$}

For this class, the existence of bound-state solutions requires $\left|g_{s}\right|>\left|g_{v}\right|$. Included into this class is the case of a pure scalar coupling, but the case of a pure vector, though, is naturally excluded. If $g_{s}<-g_{v}$ the theory is essentially relativistic. Let us define

$$
y=|x|+B / A
$$

so that (2)-(3) transmute into

$$
-\frac{\hbar^{2}}{2 m} \frac{d^{2} \psi}{d y^{2}}+\frac{1}{2} A y^{2} \psi=\left(E_{e f f}+\frac{B^{2}}{2 A}\right) \psi
$$

whose square-integrable solution satisfying the boundary conditions at $x=0$ is given by

$$
\begin{aligned}
& \psi(y)=N_{n} \exp \left(-y^{2} / 2\right) H_{n}(y), \quad n=0,1,2, \ldots \\
& E_{\text {eff }}=\left(n+\frac{1}{2}\right) \hbar \sqrt{\frac{A}{m}}-\frac{B^{2}}{2 A}
\end{aligned}
$$

where $N_{n}$ is a normalization constant and $H_{n}(y)$ is a Hermite polynomial. It follows that the KG eigenenergies are the solutions of a second-degree algebraic equation:

$$
E=-m c^{2} \frac{g_{v}}{g_{s}} \pm \frac{\left(g_{s}^{2}-g_{v}^{2}\right)^{3 / 4}}{g_{s}} \sqrt{(2 n+1) \hbar c}
$$

There is an infinite sequence of allowed KG eigenenergies with alternate parities to each sign in (13). This result clearly shows that $g_{v} \rightarrow-g_{v}$ makes $E \rightarrow-E$ without interfering in $\psi$, as stated earlier. The change $g_{s} \rightarrow$ $-g_{s}$ also makes $E \rightarrow-E$ but $\psi$ suffers a diverse sort of translation along the $x$-axis because $B$ also changes sign. Eq. (13) also shows that the KG eigenenergies are symmetrically disposed about $E=0$ in the case of a pure scalar coupling and tends to be so as $g_{s} /\left|g_{v}\right| \rightarrow \infty$.

Fig. 1 (Fig. 21) illustrates the three lowest energy levels for this class of effective potential as a function of $g_{v} / g_{s}\left(g_{s} / g_{v}\right)$. These figures show that both 
sorts of energy levels, for particles and antiparticles, are always present. It is evident from Fig. 1 that the bounded solutions for particles (antiparticles) are restricted to $E>-m c^{2}\left(E<+m c^{2}\right)$. On the other hand, from Fig. 2 one can see that the bounded solutions for particles (antiparticles) are restricted to $E>-m c^{2}\left(E<-m c^{2}\right)$ for $g_{s}>0$. Despite the particle and antiparticle energy levels share the same energy in the spectral gap between $-m c^{2}$ and $+m c^{2}$ for the case $g_{v} /\left|g_{s}\right|<1$, there is no crossing of levels.

\subsection{The effective Coulomb potential $\left(g_{s}^{2}=g_{v}^{2}\right)$}

For this class of effective potential $A=0$ and the discrete spectrum arises when $B>0$. These restrictions lead to the constraint relation

$$
E \operatorname{sgn}\left(g_{v}\right)+m c^{2} \operatorname{sgn}\left(g_{s}\right)>0
$$

Defining

$$
\begin{aligned}
& z=a|x|+b \\
& a=\left(\frac{2 m B}{\hbar^{2}}\right)^{1 / 3}, \quad b=-E_{\text {eff }} \frac{a}{B}
\end{aligned}
$$

Eq. (21) turns into the Airy differential equation

$$
\frac{d^{2} \psi}{d z^{2}}-z \psi=0
$$

which has square-integrable solutions expressed in terms of the Airy functions [30]: $\psi(z)=N \mathrm{~A}_{\mathrm{i}}(z)$, where $N$ is a normalization constant. The boundary conditions at $x=0$ lead to the quantization conditions

$$
\begin{aligned}
& \mathrm{A}_{\mathrm{i}}(b)=0, \quad \text { for odd parity solutions } \\
& \mathrm{A}_{\mathrm{i}}^{\prime}(b)=0, \quad \text { for even parity solutions }
\end{aligned}
$$

These quantization conditions have solutions only for $b<0$ and a number of them is listed are Table I [30]. One can see that demanding $b<0$ implies into an additional restriction on the KG eigenenergies: $|E|>m c^{2}$. Substitution 
of the roots of the Airy function and its first derivative into (17) allow us to obtain the energies as the solutions of a sixth-degree algebraic equation:

$$
\begin{gathered}
E^{6}-3 m^{2} c^{4} E^{4}+\left(3 m^{4} c^{6}-4|b|^{3} \hbar^{2} g_{s}^{2}\right) c^{2} E^{2} \\
-8|b|^{3} \hbar^{2} m c^{4} g_{s}^{2} \operatorname{sgn}\left(g_{s} g_{v}\right) E-\left(4|b|^{3} \hbar^{2} g_{s}^{2}+m^{4} c^{6}\right) m^{2} c^{6}=0
\end{gathered}
$$

Since there are no explicit solutions to this algebraic equation in terms of radicals, we satisfy ourselves verifying that the roots of Eq. (18) always satisfy the requirement $|E|>m c^{2}$ by using the Descartes' rule of signs (henceforth DRS). The DRS states that an algebraic equation with real coefficients $a_{k} \lambda^{k}+\ldots+a_{1} \lambda+a_{0}=0$ the difference between the number of changes of signs in the sequence $a_{k}, \ldots, a_{1}, a_{0}$ and the number of positive real roots is an even number or zero, with a root of multiplicity $k$ counted as $k$ roots and not counting the null coefficients (see, e.g., 31]). The verification of the existence of solutions for $E>m c^{2}$ is made simpler if we write $E=m c^{2}+\varepsilon$. We get

$$
\begin{gathered}
\varepsilon^{6}+6 m c^{2} \varepsilon^{5}+12 m^{2} c^{4} \varepsilon^{4}+8 m^{3} c^{6} \varepsilon^{3}-4|b|^{3} \hbar^{2} c^{2} g_{s}^{2} \varepsilon^{2} \\
-8|b|^{3} \hbar^{2} m c^{4} g_{s}^{2}\left[1+\operatorname{sgn}\left(g_{s} g_{v}\right)\right] \varepsilon-8|b|^{3} \hbar^{2} m^{2} c^{6} g_{s}^{2} \operatorname{sgn}\left(g_{s} g_{v}\right)=0
\end{gathered}
$$

Observing the difference of signs among the coefficients of the leading coefficient and the lowest degree it becomes clear that there exist positive roots for $g_{v}=g_{s}$. In this particular case the DRS assures that there exists just one solution, since there is only one change of sign in the sequence of coefficients of (19). For $g_{v}=-g_{s}$, though, the DRS implies that there exist zero or two positive roots. It is interesting to note that this result is true whatever the fermion mass and the coupling constants. The very same conclusion for $E<-m c^{2}$ can be obtained by observing that $g_{v} \rightarrow-g_{v}$ by the change $E \rightarrow-E$. However, this is not the whole story because the KG eigenenergies with $|E|>m c^{2}$ are not only given by the solutions of Eq. (18). As a matter of fact, they have also to satisfy the constraint expressed by Eq. (14). As a result, there are only KG eigenenergies with $E>m c^{2}\left(E<-m c^{2}\right)$ for $g_{v}=\left|g_{s}\right|\left(g_{v}=-\left|g_{s}\right|\right)$. This conclusion confirms what has already been mentioned in the last paragraph of the Sec. 2: the spectrum contains either particle-energy levels or antiparticle-energy levels. Furthermore, it is not difficult to see that the spectrum for the case $g_{v}=-g_{s}$ can be obtained from that for $g_{v}=g_{s}$ by changing $E$ by $-E$. 


\section{Conclusions}

In summary, we have succeed in the proposal for searching bounded solutions of the KG equation with a mixed vector-scalar Coulomb potential. An opportunity was given to analyze some aspects of the KG equation which have not been approached in the literature yet.

In general, there exist bounded solutions for particles and antiparticles. Nevertheless, for $\left|g_{s}\right|=\left|g_{v}\right|$ there are bounded solutions either for particles or antiparticles. This is a clear manifestation of the phase transition which occurs when $\left|g_{s}\right|=\left|g_{v}\right|$. The solutions of the KG equation with a Coulomb potential present a continuous transition as the ratio $g_{s} / g_{v}\left(\right.$ or $\left.g_{v} / g_{s}\right)$ varies. However, when $\left|g_{s}\right|=\left|g_{v}\right|$ the phase transition shows its face not only for the eigenenergy but also for the eigenfunction.

Finally, we draw attention to the fact that no matter how strong the potentials are, as far as $\left|g_{s}\right| \geq\left|g_{v}\right|$, the energy levels for particles (antiparticles) never dive into the lower (upper) continuum. Thus there is no room

for the production of particle-antiparticle pairs. This all means that Klein's paradox never comes to the scenario.

\section{Acknowledgments}

This work was supported in part by means of funds provided by $\mathrm{CNPq}$ and FAPESP. 


\section{References}

[1] C.J. Efthimiou, D.A. Spector, A collection of exercises in twodimensional physics, Part. I, arXiv: hep-th/0003190.

[2] S. Coleman, et al., Ann. Phys. (N.Y.) 93 (1975) 267.

[3] S. Coleman, Ann. Phys. (N.Y.) 101 (1976) 239.

[4] J. Fröhlich and E. Seiler, Helv. Phys. Acta 49 (1976) 889.

[5] A.Z. Capri and R. Ferrari, Can. J. Phys. 63 (1985) 1029.

[6] H. Galić, Am. J. Phys. 56 (1988) 312.

[7] G.'t Hooft, Nucl. Phys. B 75 (1974) 461.

[8] J. Kogut and L. Susskind, Phys. Rev. D 9 (1974) 3501.

[9] R.S. Bhalerao and B. Ram, Am. J. Phys. 69 (2001) 817.

[10] A.S. de Castro, Am. J. Phys. 70 (2002) 450.

[11] R.M. Cavalcanti, Am. J. Phys. 70 (2002) 451.

[12] J.R. Hiller, Am. J. Phys. 70 (2002) 522.

[13] W. Lucha, et al., Phys. Rep. 200 (1991) 127 and references therein.

[14] B.D. Serot, J.D. Walecka, in: J.W. Negele, E. Vogt (Eds.), Advances in Nuclear Physics, vol. 16, Plenum Press, New York, 1986.

[15] J.N. Ginocchio, Phys. Rev. Lett. 78 (1997) 436.

[16] J.N. Ginocchio, A. Leviatan, Phys. Lett. B 425 (1998) 1.

[17] J.N. Ginocchio, Phys. Rep. 315 (1999) 231.

[18] P. Alberto, et al., Phys. Rev. Lett. 86 (2001) 5015.

[19] P. Alberto, et al., Phys. Rev. C 65 (2002) 034307.

[20] T.-S. Chen, et al., Chin. Phys. Lett. 20 (2003) 358. 
[21] G. Mao, Phys. Rev. C 67 (2003) 044318.

[22] S.-G. Zhou, et al., Phys. Rev. Lett. 91 (2003) 262501.

[23] R. Lisboa, et al., Phys. Rev. C 69 (2004) 024319.

[24] J.N. Ginocchio, Phys. Rev. C 69 (2004)034318.

[25] R. Lisboa, et al., Int. J. Mod. Phys. D 13 (2004) 1447.

[26] J.-Y. Guo and Z.-Q. Siang, Phys. Lett. A 338 (2005) 90.

[27] J.-Y. Guo, et al., Nucl. Phys. A, in press.

[28] A.S. de Castro, Phys. Lett. A 305 (2002) 100.

[29] Y. Nogami, et al., Am. J. Phys. 71 (2003) 950.

[30] M. Abramowitz and I.A. Stegun, Handbook of Mathematical Functions, Dover, Toronto, 1965.

[31] I.N. Bronshtein and K.A. Semendyayev, Handbook of Mathematics, Springer-Verlag, Berlin, 1998. 
Table 1: The first roots of the Airy function and its first derivative

\begin{tabular}{cc}
\hline$A i^{\prime}(-|b|)=0$ & $A i(-|b|)=0$ \\
\hline 1.01879 & 2.33810 \\
3.24819 & 4.08794 \\
4.82009 & 5.52055 \\
\hline
\end{tabular}

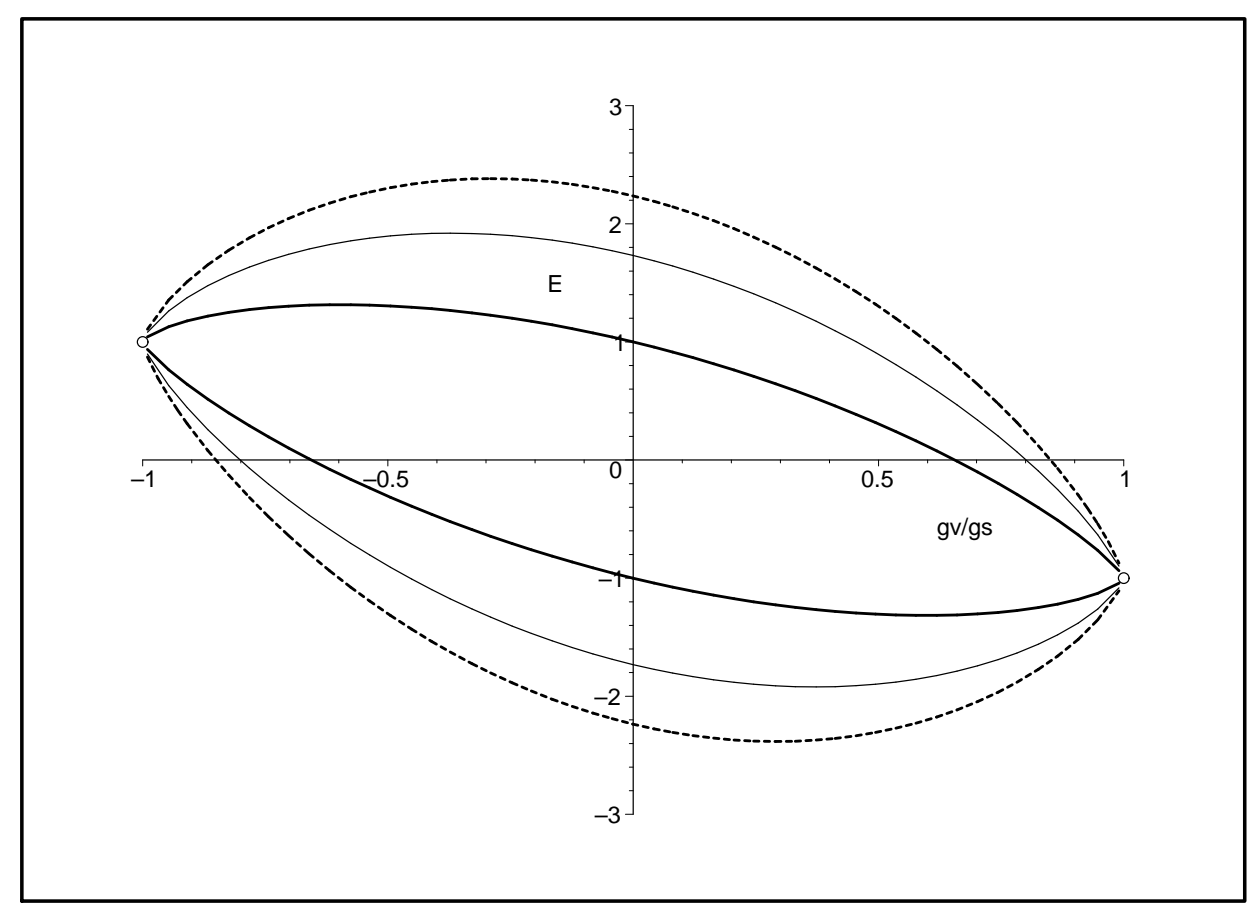

Figure 1: KG eigenenergies for the three lowest energy levels as a function of $g_{v} / g_{s}$ for $g_{s}=1$. The full thick line stands for $n=0$, the full thin line for $n=1$ and the dashed line for $n=2(m=\hbar=c=1)$. 


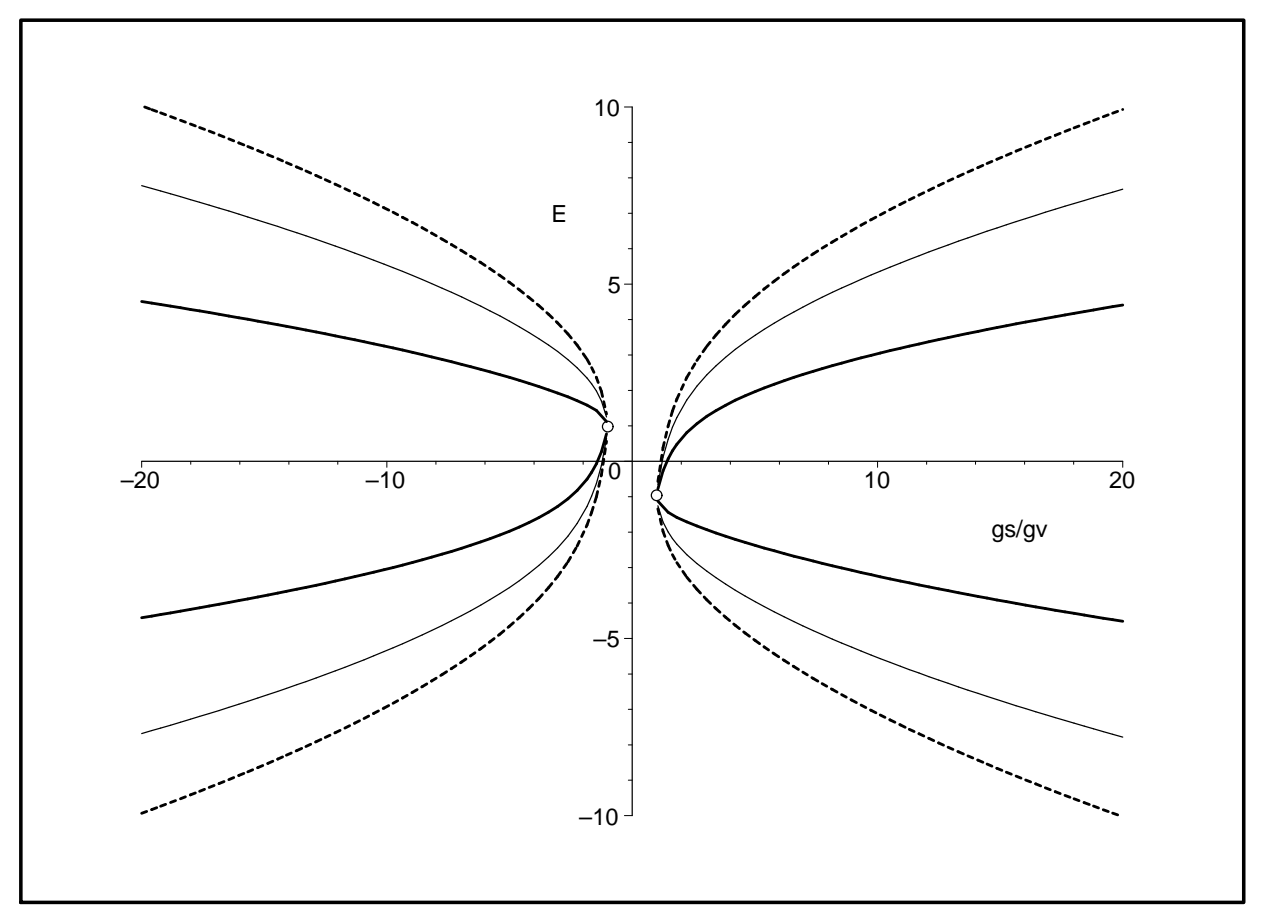

Figure 2: The same as in Fig. 1 as a function of $g_{s} / g_{v}$ for $g_{v}=1$. 